\title{
Phase Sensitivity of Synaptic Modifications in Oscillating Cells of Rat Visual Cortex
}

\author{
Valérie Wespatat, Frank Tennigkeit, and Wolf Singer \\ Department of Neurophysiology, Max-Planck-Institute for Brain Research, D-60528 Frankfurt/Main, Germany
}

Synaptic modifications depend on the amplitude and temporal relations of presynaptic and postsynaptic activation. The interactions among these variables are complex and hard to predict when neurons engage in synchronized high-frequency oscillations in the $\beta$ and $\gamma$ frequency range, as is often observed during signal processing in the cerebral cortex. Here we investigate in layer II/III pyramidal cells of rat visual cortex slices how synapses change when synchronized, oscillatory multifiber activity impinges on postsynaptic neurons during membrane potential $\left(V_{\mathrm{m}}\right)$ oscillations at 20 and $40 \mathrm{~Hz}$. Synapses underwent long-term potentiation (LTP) when EPSPs coincided with the peaks of the $V_{\mathrm{m}}$ oscillations but exhibited long-term depression (LTD) when EPSPs coincided with the troughs. The induction of LTP but not of LTD was NMDA receptor dependent, required additional activation of muscarinic receptors in older animals, and persisted in a kainate-driven increased conductance state. Thus, even when neuronal networks engage in high-frequency oscillations, synaptic plasticity remains exquisitely sensitive to the timing of discharges. This is an essential prerequisite for theories which assume that precise synchronization of discharges serves as signature of relatedness in distributed processing.

Key words: cortex; EPSP; excitatory postsynaptic; neuromodulation; oscillator; slice; plasticity

\section{Introduction}

In numerous structures of the nervous system and in particular in the cerebral cortex, neuronal activity is often characterized by an oscillatory patterning in the $\beta$ - and $\gamma$-frequency range $(20-60$ $\mathrm{Hz}$ ) that is associated with precise synchronization of discharges (Silva et al., 1991; Singer, 1993, 1999; Buzsaki and Chrobak, 1995; Steriade, 1999; Tallon-Baudry and Bertrand, 1999). Evidence suggests that this synchronization serves response selection in the context of various cognitive functions such as binding, perceptual rivalry, attention, and short- as well as long-term memory (Fernandez et al., 1999; Gray, 1999; Miltner et al., 1999; Singer, 1999; Tallon-Baudry and Bertrand, 1999; Engel et al., 2001; Fries et al., 2001; Parkhurst et al., 2002). The likely mechanism is that coincident EPSPs summate more effectively in target structures than temporally dispersed EPSPs (Usrey and Reid, 1999). This enhanced saliency predisposes synchronized responses for further joint processing.

Temporal relations among the discharges of interconnected neurons are also an important variable in synaptic plasticity. Synapses undergo long-term potentiation (LTP) when the EPSP precedes the postsynaptic spike within a few tens of milliseconds, and they undergo long-term depression (LTD) when the EPSP follows the spike [spike time-dependent plasticity (STDP)].

Received June 8, 2004; revised Aug. 27, 2004; accepted Aug. 27, 2004.

This study was supported by grants from the Max-Planck-Society, the Deutsche Forschungsgemeinschaft (SFB 269), and the Körber Foundation. We thank M. Duesmann, P. Janson, R. Stiller, and S. Völsing for technical assistance and J. Eilers and K. Stiefel for comments on this manuscript. We also give special thanks to K. Stiefel for the custom-made Labview analysis tools.

Correspondence should be addressed to Wolf Singer, Department of Neurophysiology, Max-Planck-Institute fur Hirmforschung, Deutschordenstrasse 46, D-60528 Frankfurt/Main, Germany. E-mail: singer@mpih-frankfurt.mpg.de.

DO1:10.1523/JNEUROSCI.2221-04.2004

Copyright $\odot 2004$ Society for Neuroscience $\quad 0270-6474 / 04 / 249067-09 \$ 15.00 / 0$
Thus, synapses strengthen activity that is causally related to postsynaptic firing (Markram et al., 1997; Egger et al., 1999; Feldman, 2000; Sjöström et al., 2001; Froemke and Dan, 2002).

Interactions between presynaptic and postsynaptic activity become hard to predict when neuronal networks engage in highfrequency oscillations, as is often the case in vivo. Previous studies on the phase sensitivity of synaptic modifications have investigated $\theta$ oscillations $(7 \mathrm{~Hz}$ ) in the hippocampus and relied on field potential recordings for both the assessment of oscillation phase and synaptic modifications. These studies demonstrated LTP of field EPSPs when pairing afferent volleys with the depolarizing phases of the oscillations and a resetting of LTP when applying the stimuli during the hyperpolarizing phases (Huerta and Lisman, 1993, 1995; Hölscher et al., 1997). At these low frequencies, one expects little interference between successive activation cycles because STDP effects are weak when intervals between EPSPs and postsynaptic spikes exceed $100 \mathrm{msec}$ (Markram et al., 1997; Egger et al., 1999; Feldman, 2000; Sjöström et al., 2001; Froemke and Dan, 2002), as is the case with $\theta$ oscillations. Thus, synaptic modifications are determined essentially by the level of membrane potential $\left(V_{\mathrm{m}}\right)$ depolarization at the time of EPSP arrival. Conditions become much less predictable at oscillation frequencies in the $\beta$ and $\gamma$ range that are common in the cerebral cortex. Here, even at phase offsets of $180^{\circ}$, EPSPs precede and follow postsynaptic spikes at intervals $\leq 25 \mathrm{msec}$, and thus STDP effects can be expected to cause strong interference between successive cycles. Furthermore, because discharges are periodic in oscillating networks, phase shifts can lead to ambiguous timing relations between presynaptic and postsynaptic firing. Such phase shifts are frequent and commonly observed in multisite recordings from the cerebral cortex (Varela et al., 2001). With $180^{\circ}$ phase shifts, EPSPs are equidistant to preceding and following spikes, 
and in this case no clear predictions follow from STDP rules, provided that oscillation frequencies are high enough to bring EPSPs sufficiently close to the respective preceding and following spikes. With $\beta$ and $\gamma$ oscillations, these intervals are $<25 \mathrm{msec}$ and hence well within the critical range of STDP.

These considerations call for an experimental answer to the question of whether and how synapses change in networks that engage in high-frequency oscillations and whether the modifications remain sensitive to the precise timing relations between presynaptic and postsynaptic discharges. Clarification of this question is crucial in the context of the hypothesis that precise synchronization of discharges serves response selection and the definition of relations in signal processing.

\section{Materials and Methods}

All experiments were performed in accordance with European laws and National Institutes of Health guidelines for the treatment of animals in research.

Slice preparation. Cortical tissue was obtained from 3- to 5-week-old [postnatal day 18-33 (P18-33), P22 \pm 4 ; mean \pm SD] Wistar rats under deep halothane or isoflurane anesthesia. In a subset of experiments (13 cells), slices were taken from 2-week-old rats (P12-14, P13 \pm 1 ). Coronal slices $(300-400 \mu \mathrm{m})$ of primary visual cortex were prepared in ice-cold low-calcium artificial CSF (ACSF) containing (in mM): either $125 \mathrm{NaCl}$, $2.5 \mathrm{KCl}, 1.25 \mathrm{NaH}_{2} \mathrm{PO}_{4}, 25 \mathrm{NaHCO}_{3}, 1 \mathrm{CaCl}_{2}, 2 \mathrm{MgCl}_{2}, 25$ glucose or, alternatively, $87 \mathrm{NaCl}, 4 \mathrm{KCl}, 1.25 \mathrm{NaH}_{2} \mathrm{PO}_{4}, 25 \mathrm{NaHCO}_{3}, 0.5 \mathrm{CaCl}_{2}, 7$ $\mathrm{MgCl}_{2}, 10$ glucose, 75 saccharose, saturated with $5 \% \mathrm{CO}_{2-} 95 \% \mathrm{O}_{2}$. Slices were then transferred for $30 \mathrm{~min}$ to warm $\left(37^{\circ} \mathrm{C}\right)$ ACSF and then stored at room temperature. For recording, slices were transferred to a submersion chamber and perfused at $5 \mathrm{ml} / \mathrm{min}$ at $36^{\circ} \mathrm{C}$ with ACSF of slightly different composition (in mM): $125 \mathrm{NaCl}, 2.5 \mathrm{KCl}, 1.25 \mathrm{NaH}_{2} \mathrm{PO}_{4}, 25$ $\mathrm{NaHCO}_{3}, 2 \mathrm{CaCl}_{2}, 1 \mathrm{MgCl}_{2}, 25$ or 10 glucose.

Recordings. Whole-cell patch-clamp recordings were performed on 201 layer II/III pyramidal neurons in the current-clamp mode (Axoclamp 2B, Axon Instruments, Foster City, CA). Patch electrodes (5-10 $\mathrm{M} \Omega$ in ACSF) were filled with intracellular solution. Solution 1 contained (in mM): $100 \mathrm{~K}$-gluconate, $20 \mathrm{KCl}, 4 \mathrm{ATP}-\mathrm{Mg}^{2+}, 10$ phosphocreatine, $0.3 \mathrm{Na}^{+}$-GTP, 10 HEPES. Solution 2 contained (in $\mathrm{mm}$ ): 130 K-gluconate, $10 \mathrm{KCl}, 3 \mathrm{ATP}-\mathrm{Mg}^{2+}, 10$ phosphocreatine, $0.3 \mathrm{Na}^{+}$-GTP, 10 HEPES, and creatine phosphokinase (50,000 U/l). Neurobiotin $(0.5 \%)$ was added to the intracellular solution for subsequent anatomical reconstruction. Pyramidal neurons were identified by means of differential interference contrast-infrared microscopy and characterized electrophysiologically as regular firing neurons. They had overshooting action potentials, a resting membrane potential of $-74 \pm 7 \mathrm{mV}$, a firing threshold of $-40 \pm 5 \mathrm{mV}$, and a membrane resistance of $140 \pm 61 \mathrm{M} \Omega$ (mean $\pm \mathrm{SD}$ ). Input resistance was monitored throughout the experiment by measuring voltage responses to hyperpolarizing current pulses $(-40 \mathrm{pA}, 300 \mathrm{msec})$. Only cells with stable input resistance $( \pm 20 \%)$ were analyzed. Series resistance (10-20 M $\Omega$ ) was compensated for by adjusting the bridge, and unstable recordings were discarded.

Stimulation. Horizontal afferents were stimulated extracellularly with a concentric bipolar microelectrode positioned in layer II/III, 0.5-1 mm apart from the recorded neuron. Test stimuli consisted of trains of four pulses (pulse duration $0.1 \mathrm{msec}$; interpulse interval $50 \mathrm{msec}$; intensity $2-25 \mathrm{~V}$ ), that were repeated at intervals of $10 \mathrm{sec}$. Once they had become stable, test responses were recorded for at least $10 \mathrm{~min}$ before the onset of conditioning and for up to $60 \mathrm{~min}$ after conditioning. Because of the relatively large distance between the recorded neuron and the stimulation site, monosynaptic inhibitory GABAergic PSPs were not evoked, because PSPs were completely blocked by coapplication of CNQX (20 $\mu \mathrm{M})$ and $\mathrm{APV}(50 \mu \mathrm{M})(n=14$ of 14 ; data not shown); however, we cannot exclude activation of polysynaptic GABAergic PSPs during highfrequency stimulation.

Oscillation pairing. Suprathreshold membrane potential oscillations $( \pm 10 \mathrm{mV}, 20 \mathrm{~Hz}$ and $40 \mathrm{~Hz}$ ) were evoked by sinusoidal current injection from a depolarized holding potential $(-55$ to $-40 \mathrm{mV})$, as is common in vivo (Gray and McCormick, 1996; Steriade et al., 1998; Azouz and Gray, 2000), and paired with $20 \mathrm{~Hz}$ EPSP trains. Phase angles were adjusted by imposing subthreshold oscillations at resting potential to avoid induction of synaptic plasticity during the adjustment. Stimulus sequences were timed individually as a function of the EPSP rise time (onset to peak, $7 \pm 2 \mathrm{msec}$ ) so that EPSP peaks coincided with either the peaks or the troughs of the $V_{\mathrm{m}}$ oscillations (see Fig. 1). With peak pairing, EPSP onsets preceded oscillation peaks and the concomitant spike by $10 \pm 3 \mathrm{msec}(20$ $\mathrm{Hz}$ oscillations) or $9 \pm 2 \mathrm{msec}$ ( $40 \mathrm{~Hz}$ oscillations) and followed the discharges of the preceding cycles by $40 \pm 3 \mathrm{msec}(20 \mathrm{~Hz})$ or $16 \pm 2 \mathrm{msec}$ $(40 \mathrm{~Hz})$. Because of the latency jitter of oscillation-evoked spikes and variable EPSP rise times, the EPSP peaks coincided only on average with the spikes but in individual cases could either precede or follow spike onset with an uncontrollable scatter of \pm 2 msec. With trough pairing, EPSP onsets lagged oscillation peaks and concomitant spikes by $13 \pm 3$ $\operatorname{msec}(20 \mathrm{~Hz})$ and $5 \pm 2 \mathrm{msec}(40 \mathrm{~Hz})$ and preceded the discharges of the subsequent peak by $37 \pm 3 \mathrm{msec}(20 \mathrm{~Hz})$ or $20 \pm 2 \mathrm{msec}(40 \mathrm{~Hz})$. For conditioning, sequences of 10 oscillation cycles were applied at $1 \mathrm{sec}$ intervals and repeated until 100 EPSPs had been delivered, i.e., 10 times for $20 \mathrm{~Hz}$ oscillations and 20 times for $40 \mathrm{~Hz}$ oscillations. Occasionally a depolarizing cycle failed to evoke a spike discharge, but there was no significant difference in the number of spikes evoked during peak and trough pairing, respectively ( $p>0.05$; ANOVA).

Evaluation of synaptic plasticity. The four test EPSPs were recorded continuously before and after pairing. We used repetitive stimulation (four test EPSPs) to be able to assess a potential redistribution of synaptic efficacy (Markram and Tsodyks, 1996). To this end, the amplitude changes of the first, second, third, and fourth EPSP of the test response were determined. In the majority of cases (193 of 201 cells), the polarity of modifications was similar for all four EPSPs, and a redistribution of synaptic amplitudes occurred in only eight cells. Therefore, we only used amplitude changes of the first EPSP for the quantification of synaptic modifications.

We routinely measured and plotted several EPSP parameters: EPSP amplitude, EPSP time to peak, EPSP area under the curve, and EPSP slope. Because EPSP slope, amplitude, and area changed in exactly the same way, we opted for the EPSP amplitude for the assessment of synaptic changes (supplemental Fig. 1; supplemental material, available at www.jneurosci.org).

EPSP amplitudes were compared during epochs of equal length, one immediately before and the other after the pairing by 15-20 min, with each epoch including 20 normalized and averaged data points. Changes were classified as LTP or LTD if they were significant at the $p<0.05$ level (paired Student's $t$ test). In experiments during which kainate was washed in and out, baseline EPSPs preceding kainate application were compared with EPSPs after the pairing by 35-40 min. Pairwise differences between protocols were tested with the Mann-Whitney rank sum test unless stated otherwise. We opted for the nonparametric MannWhitney rank sum test to avoid false assumptions about the distribution of the data. In addition we examined the significance of changes for each experimental protocol by computing grand averages. We considered all cells in this calculation as well, including those in which changes had not reached significance, to avoid selection of only those cells showing strong effects.

Pharmacology. Almost all drugs were bath applied and present throughout the experiments. The cholinergic agonist carbachol $(\mathrm{CCH})$ $(5 \mu \mathrm{M})$ was always coapplied with the antagonist gallamine $(50 \mu \mathrm{M})$ to avoid depression of release (Bröcher et al., 1992). This allowed us to start experiments with middle-sized EPSPs $(\sim 3 \mathrm{mV})$ potentially able to undergo both LTP and LTD without having to recruit a larger synaptic circuit by simply increasing the stimulation intensity. Effects of $\mathrm{CCH}$ wash-in were monitored in a subset of cells. $\mathrm{CCH}$ application led to a slight $V_{\mathrm{m}}$ depolarization ( +3 to $+8 \mathrm{mV} ; n=16$ cells) and occasionally shortened interspike intervals in response to depolarizing current steps, increasing the number of spikes generated by a constant current pulse by a factor of $1.58(n=7)$. $\mathrm{CCH}$ had no measurable effect on input resistance (106 $\pm 6 \% ; n=9$ cells). After repetitive spiking, we sporadically observed an afterdepolarization of +1.0 to $+2.5 \mathrm{mV}(n=3)$ that lasted $\sim 1000$ msec. Application of CCH had no effect on the incidence of spikes 
evoked during pairing ( $p>0.5$; Mann-Whitney rank sum test, compared with pairing in ACSF), nor did $\mathrm{CCH}$ induce population oscillations in the slice.

The NMDA-receptor blocker APV $(50 \mu \mathrm{M})$ was added to the ACSF alone or in addition to carbachol-gallamine. The muscarinic receptor antagonist scopolamine $(10 \mu \mathrm{M})$ and the nicotinic receptor antagonist hexamethonium $(100 \mu \mathrm{M})$ either were separately applied in addition to carbachol-gallamine or were coapplied in ACSF. The muscarinic agonist muscarine $(20 \mu \mathrm{M})$ was added to the ACSF.

Although carbachol was present throughout the experiments, kainate (200 nM) was washed in just before and washed out immediately after pairing. The application of this low dose of kainate did not remarkably alter the $V_{\mathrm{m}}$ of the cells (slight depolarization of $2 \mathrm{mV}$ on average) but resulted in a drop of input resistance $(-16 \pm 4 \% ; n=12$ cells) associated with decreased EPSP amplitudes ( $-16 \pm 7 \% ; n=12$ cells) and increased synaptic background activity (data not shown). Furthermore, kainate accelerated EPSP decay and reduced EPSP summation (see Fig. 6, inset).

In six experiments, postsynaptic $\mathrm{Ca}^{2+}$ was buffered with BAPTA (10 $\mathrm{mm}$ ) in the intracellular solution.

\section{Results}

We examined synaptic plasticity after synchronized $\beta$ and $\gamma$ oscillations in rat visual cortex slices. Slices were taken mainly from juvenile to adult rats (P18-33) and analyzed at close to physiological temperature $\left(36^{\circ} \mathrm{C}\right)$. Inhibition was not antagonized, extracellular $\left[\mathrm{Ca}^{2+}\right.$ ] was set to $2 \mathrm{~mm}$, holding $V_{\mathrm{m}}$ was maintained at levels characteristic of "up-states" (Cossart et al., 2003; Shu et al., 2003), the amplitude of the imposed $V_{\mathrm{m}}$ oscillations was in the same range as observed during $\gamma$ oscillations in vivo (Azouz and Gray, 2000), and compound multifiber EPSPs, as are likely to occur when afferent cells synchronize their discharges, were evoked.

Layer II/III pyramidal cells recorded in whole-cell configuration were forced to discharge at 20 or $40 \mathrm{~Hz}$ by injection of sinusoidally modulated currents. For the induction of synaptic modifications, we paired these oscillatory, high-frequency responses with trains of synchronized multifiber EPSPs that oscillated at 20 $\mathrm{Hz}$ and were evoked by electrodes placed laterally to the recorded cells. Stimulus trains were timed so that the peaks of the EPSPs were synchronized with either the depolarizing (peak pairing) or hyperpolarizing maxima (trough pairing) of the membrane potential oscillations (Fig. 1a,b). Synaptic modifications were assessed by comparing the amplitudes of the first of four EPSPs (responses to four pulses separated by $50 \mathrm{msec}$, repeated every 10 $\mathrm{sec}$ ) before and after pairing (Student's $t$ test; see Materials and Methods) (Fig. 1c,d).

\section{Age dependence}

Because many studies on STDP have been performed in young animals (Markram et al., 1997; Egger et al., 1999), we first ran a series of experiments with slices of 2-week-old rats (P13 slices; $n=13$; see Materials and Methods), activating presynaptic fibers and postsynaptic cells at $20 \mathrm{~Hz}$. Peak pairing led to significant changes in three of six experiments, all of which were LTP, whereas trough pairing led to significant changes in four of seven experiments, all of which consisted of LTD (Fig. 2a). These effects remained significant in the grand averages, in which also those cells were included in which modifications had not reached significance (peak: $122 \pm 32 \%$, mean $\pm \mathrm{SD}, n=6, p<0.005$; trough: $76 \pm 20 \%, n=7, p<0.005$ ) (Fig. $2 b$ ). Thus, in young rats, the polarity of synaptic modifications was sensitive to the phase relation between sustained presynaptic and postsynaptic discharge sequences when both oscillated at $20 \mathrm{~Hz}$.

Evidence indicates that plasticity thresholds rise with age be-

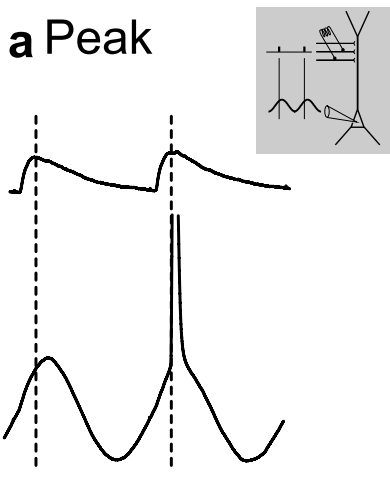

b Trough

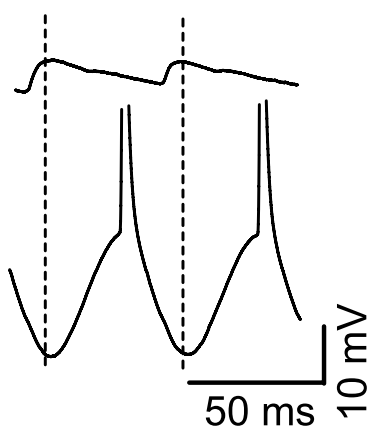

C Peak oscillation pairing $\mathbf{d}$ Trough oscillation pairing

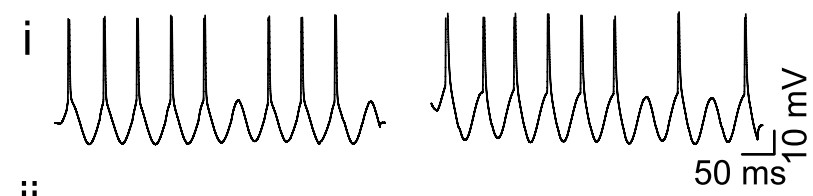

ii
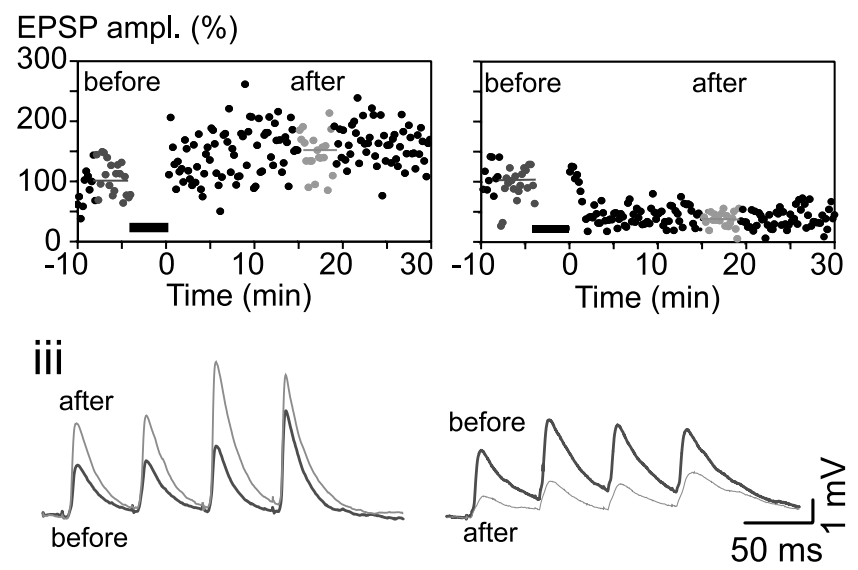

Figure 1. Oscillation pairing protocols and examples of resulting synaptic modifications. Gray window shows schematic illustration of pairing configuration. Extracellular stimulation at $20 \mathrm{~Hz}$ in layer II/III was paired with injection of suprathreshold sinusoidal $(20 \mathrm{~Hz})$ current into the soma of layer II/III pyramidal neurons. $a$, Peak pairing configuration. EPSPs (top) coincide with the depolarizing peaks of $V_{\mathrm{m}}$ oscillations and the concomitant spikes (bottom). $b$, Trough pairing configuration. EPSPs (top) coincide with the hyperpolarizing troughs of $V_{m}$ oscillations (bottom). c, Example of peak pairing-induced LTP. d, Example of trough pairing-induced LTD. $i$, Postsynaptic responses during peak and trough pairing. ii, Time course of first EPSP amplitude changes (each point corresponds to a single response normalized to baseline; gray horizontal lines indicate intervals during which EPSPs shown in iii were averaged; thick bar indicates time of pairing). iii, Averaged EPSPS ( $n=20$ ) measured during 5 min before (black line) and 15-20 min after pairing (thin line).

cause of increasing inhibition (Kato et al., 1991) and because of a developmental switch in NMDA receptor subunits (Hestrin, 1992; Nase et al., 1999; Quinlan et al., 1999). To assess whether the observed phase sensitivity persists in the more mature cortex, we repeated experiments in older animals (3-5 weeks; see Materials and Methods). Because we observed no differences among the results of this age group, we pooled the respective data and for simplicity address this age group as P22. In P22 slices, peak pairing no longer caused LTP. Significant modifications were observed in 7 of 16 experiments, and these consisted of LTD. Trough pairing led to similar results as in the younger rats. It led to significant modifications in five of six cells, and these were all LTD (Fig. 2c). In the grand averages, both the peak- and trough 

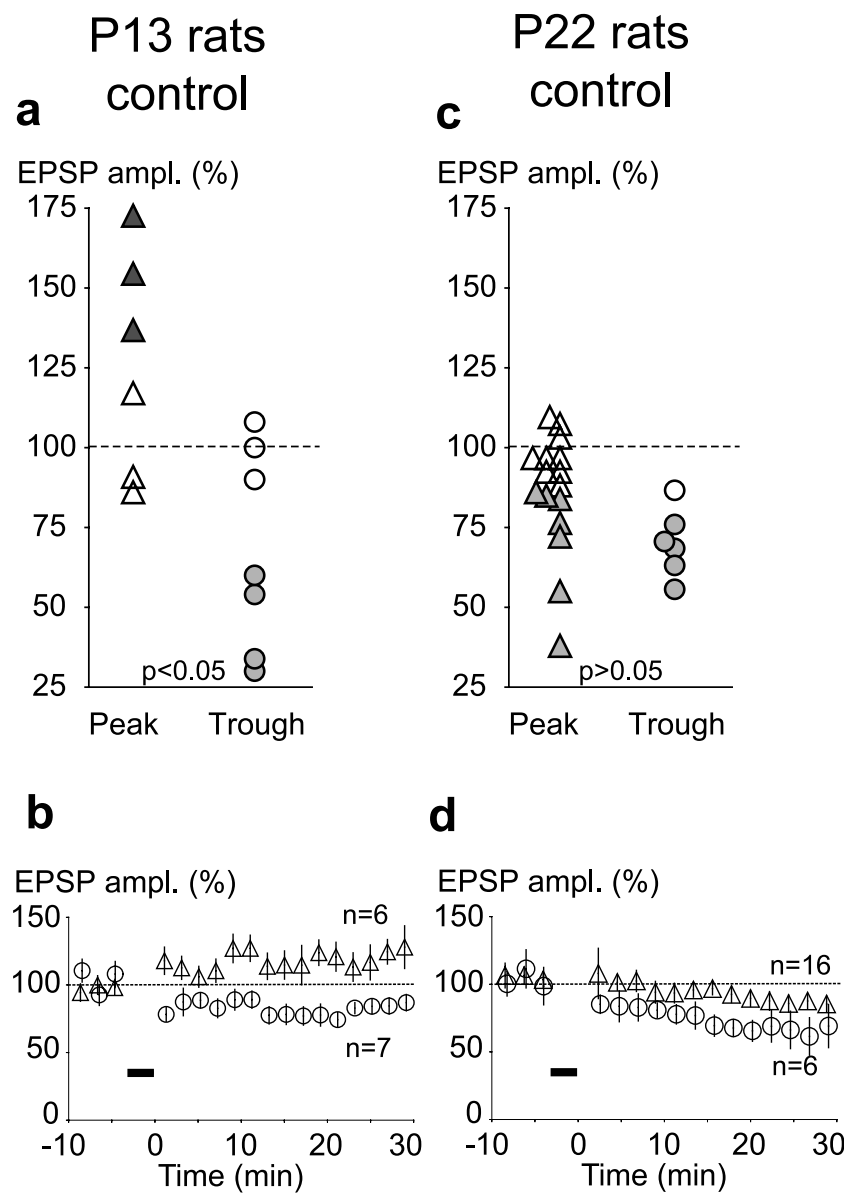

\section{e}

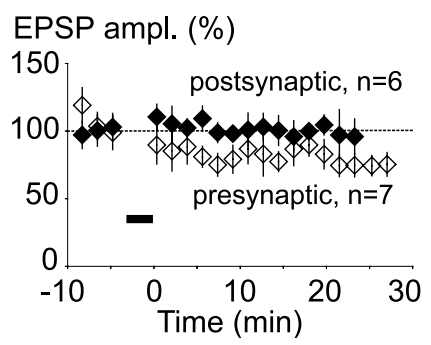

Figure 2. Phase sensitivity of synaptic modifications during sustained high-frequency oscillations depends on age. $a$, Synaptic modifications after peak and trough pairing in P13 rats in control ACSF. Each symbol corresponds to one experiment and represents the pairing-induced change of the first test EPSP averaged across 20 measurements taken $5 \mathrm{~min}$ before and $15-20$ min after pairing. Black symbols represent significant LTP, gray symbols represent significant LTD, and empty symbols represent no significant changes. $p$ value refers to the difference between peak and trough pairing (Mann-Whitney rank sum test for all experiments). $b$, Time course of EPSP amplitude changes (grand averages) from the same experiments as in $a$. Symbols represent EPSP amplitudes (mean \pm SEM) normalized and averaged across cells and plotted at $2 \mathrm{~min}$ intervals. Triangles indicate peak pairing; circles indicate trough pairing; horizontal bar indicates pairing interval. c, Synaptic modifications after peak and trough pairing in P22 rats in control ACSF. Symbols and statistics as in $a$. $d$, Time course of EPSP amplitude changes (grand averages) from the same experiments as in c (mean \pm SD). e, Synaptic modifications after either postsynaptic (filled symbols) or presynaptic (empty symbols) stimulation alone in $\mathrm{P} 22$ rats.

pairing-induced LTD was significant (peak: $87 \pm 6 \%, n=16, p<$ 0.001; trough: $66 \pm 8 \%, n=6, p<0.001$ ) (Fig. $2 d$ ). Thus, in older rats, synaptic modifications were no longer phase sensitive and consisted exclusively of LTD.

Both LTP and LTD can be induced in the absence of presyn- aptic stimulation if the postsynaptic neuron is strongly activated by current injection (Alonso et al., 1990; Volgushev et al., 1997) or antidromic stimulation (Christofi et al., 1993). To test whether nonassociative mechanisms had contributed to LTD induction, we performed six experiments in P22 slices, with postsynaptic suprathreshold $20 \mathrm{~Hz} V_{\mathrm{m}}$ oscillations, but without evoking EPSPs. In none of the experiments did postsynaptic stimulation alone produce significant changes of EPSPs (grand average, $99 \pm$ $10 \% ; n=6 ; p>0.1$ ) (Fig. $2 e$ ). This result excludes the possibility that LTD is caused simply by rundown of the preparation in older slices. In an additional seven experiments, we applied presynaptic stimulation without postsynaptic current injection and thus without spike discharges. Presynaptic stimulation alone evoked LTD in four of seven cells (grand average, $85 \pm 10 \% ; n=7 ; p<$ 0.001 ) (Fig. 2e). Thus, uncorrelated sustained oscillatory presynaptic activity resulted in a nonassociative synaptic depression.

\section{Dependence on cholinergic modulation}

During arousal, cortical networks are exposed to sustained release of acetylcholine (ACh) (Celesia and Jasper, 1966; Marrosu et al., 1995), and activation of muscarinic receptors has been identified as a necessary prerequisite for the occurrence of $\gamma$ oscillations and spike synchronization in vivo (Rodriguez et al., 2001). Furthermore, acetylcholine has been reported to facilitate LTP (Bröcher et al., 1992). Therefore, we wondered whether we could rescue the LTP observed in P13 slices in older slices by bath applying the cholinergic agonist carbachol ( $5 \mu \mathrm{M} ; n=22)$. In carbachol-treated P22 slices, peak pairing caused slight but significant LTP in 7 of 14 cells, whereas trough pairing caused significant LTD in 6 of 8 cells (Fig. $3 a$ ). These modifications also remained significant in the grand averages that also included the cells in which modifications had not reached significance (peak: $111 \pm 11 \%, n=14, p<0.001$; trough: $72 \pm 9 \%, n=8, p<0.001$ ) (Fig. $3 b$ ). Thus, in the presence of carbachol, results resembled those obtained in P13 slices in control ACSF, i.e., the polarity of synaptic modifications was sensitive to the phase relation between sustained presynaptic and postsynaptic $\beta$ oscillations.

To examine whether the differences between peak and trough pairing persisted also at higher oscillation frequencies, we performed additional experiments using carbachol, now driving the postsynaptic cell at $40 \mathrm{~Hz}(n=22)$. These experiments were also performed in P22 slices. We kept presynaptic stimulation at 20 $\mathrm{Hz}$ to avoid high-frequency attenuation of EPSPs (Deuchars et al., 1994; Markram and Tsodyks, 1996) and to mimic typical in vivo conditions. In fact, when cortical neurons engage in $\gamma$ oscillations in vivo, their discharges tend to skip cycles (Gray and McCormick, 1996; Azouz and Gray, 2000) and usually occur at lower frequencies than the $V_{\mathrm{m}}$ oscillations. At $40 \mathrm{~Hz}$, peak pairing led to significant modifications in 5 of 11 experiments, all consisting of LTP, whereas trough pairing led to significant changes in 7 of 11 slices, all consisting of LTD (Fig. 3c). Both modifications remained significant in the grand averages (peak: $110 \pm$ $12 \%, n=11, p<0.005$; trough: $78 \pm 16 \%, n=11, p<0.001$ ) (Fig. $3 d$ ). These results are similar to those found with $20 \mathrm{~Hz}$ oscillations and indicate that synaptic modifications remain exquisitely phase sensitive even during sustained $\gamma$ oscillations.

\section{Synergy between muscarinic and NMDA receptors}

The high-frequency stimuli applied to layer II/III may have coactivated cholinergic afferents. To examine this possibility and assess plasticity in the absence of any cholinergic effects, we bath applied the muscarinic antagonist scopolamine $(10 \mu \mathrm{M})$ in combination with the nicotinic antagonist hexamethonium $(100 \mu \mathrm{M})$ 

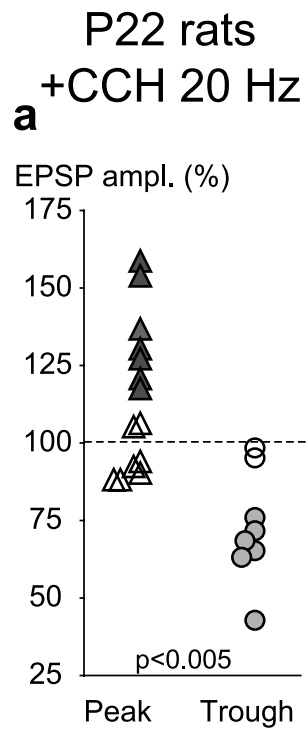

b
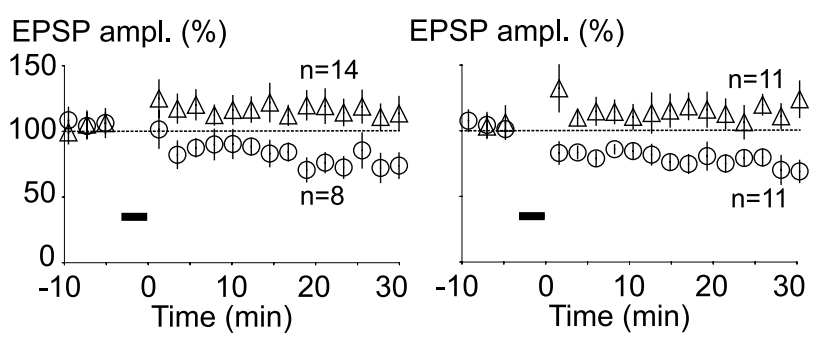

Figure 3. Phase sensitivity of synaptic modifications during sustained high-frequency oscillations is restored by cholinergic receptor activation in older rats at both 20 and $40 \mathrm{~Hz} . a, b, \mathrm{P} 22$ rats in $\mathrm{CCH}$ with $20 \mathrm{~Hz}$ oscillations. $a$, Synaptic modifications after peak and trough pairing. Symbols are the same as in Figure 2a. b, Corresponding time course of EPSP amplitude changes (mean \pm SD). Symbols are the same as in Figure $2 b . C, d, P 22$ rats in $C \mathrm{CH}$ with $40 \mathrm{~Hz}$ oscillations. $c$, Synaptic modifications after peak and trough pairing. $d$, Corresponding time course of EPSP amplitude changes (mean \pm SD).

in 15 experiments performed with $20 \mathrm{~Hz}$ modulation. Peak and trough pairing led to significant modifications in eight of nine and in five of six experiments, respectively; all of these significant changes consisted of LTD (Fig. $4 a$ ). In the grand averages, the effects of both peak and trough pairing were significant (peak: $68 \pm 7 \%, n=9, p<0.001$; trough: $76 \pm 13 \%, n=6, p<0.001$ ) (Fig. $4 d$ ). Thus, with cholinergic blockers the effects of peak and trough pairing were nearly identical and resembled closely those obtained in control ACSF, excluding a major effect of residual ACh release in control solution.

To identify the nature of the cholinergic receptors that favored LTP with peak pairing, either we blocked muscarinic receptors with scopolamine $(10 \mu \mathrm{M})$ and bath applied carbachol $(5 \mu \mathrm{M})$ to activate nicotinic receptors or we blocked nicotinic receptors with hexamethonium $(100 \mu \mathrm{M})$ and bath applied carbachol (5 $\mu \mathrm{M})$ to activate muscarinic receptors. Under muscarinic receptor blockade, peak pairing caused significant changes in six of eight experiments, five consisting of LTD and one of LTP (Fig. 4b). In the grand average, the net effect was a slight but significant LTD (94 $\pm 9 \% ; n=8 ; p<0.05)$ (Fig. 4e). Under nicotinic receptor blockade, peak pairing caused significant changes in two of six experiments, two consisting of LTP (Fig. $4 b)$. The LTP remained significant in the grand average $(112 \pm 17 \% ; n=6 ; p<0.05)$ (Fig. 4e). Thus, the cholinergic facilitation of LTP with peak pair- ing appeared to be mediated by muscarinic receptors. To confirm these findings, we performed additional experiments, now bath applying the muscarinic agonist muscarine $(20 \mu \mathrm{M})$ instead of carbachol. Under muscarinic receptor activation, peak pairing led to significant changes in four of seven cells, all consisting of LTP, whereas trough pairing resulted in LTD (four cells), LTP (two cells), and no significant change (one cell; $n=7$ ) (Fig. 4c,f). In the grand averages, synaptic modifications remained phase sensitive, with peak pairing leading to LTP $(121 \pm 20 \%$; $p<$ $0.001)$ and trough pairing leading to LTD $(85 \pm 13 \% ; p<0.001)$. Thus, LTP facilitation occurred via muscarinic receptor activation.

Depending on the stimulation protocol, LTP and LTD induction may or may not require NMDA receptor activation (Artola and Singer, 1987; Bear and Kirkwood, 1993). To determine the involvement of NMDA receptors, we bath applied the NMDA receptor antagonist APV $(50 \mu \mathrm{M})$ in P22 experiments performed at $20 \mathrm{~Hz}$ in control solution $(n=12)$ or with carbachol added $(n=6)$. APV had no effect (Fig. $5 a)$ in control solution. As in the previous experiments performed in P22 slices, both peak and trough pairing produced LTD (grand average, peak: $79 \pm 8 \%$, $n=6, p<0.001$; trough: $90 \pm 12 \%, n=6, p<0.005$ ) (Fig. $5 c$ ). These results were similar to those obtained in P22 slices in control solution $(p>0.5)$ for the outcome of peak and trough pairings, respectively. This indicates that neither peak- nor trough pairing-induced LTD was NMDA receptor dependent and complements our finding that presynaptic stimulation alone was sufficient to induce LTD (Fig. 2e). In contrast, APV affected the outcome of peak pairing in carbachol by abolishing the expected LTP. When NMDA receptors were blocked in the presence of carbachol, peak pairing induced significant modifications in three of six slices (Fig. $5 b$ ), and all of these changes consisted of LTD (grand average: $88 \pm 11 \% ; n=6 ; p<0.001$ ) (Fig. $5 c$ ). This indicates that the LTP obtained with peak pairing was NMDA receptor dependent.

Because LTD was found to be NMDA receptor independent, however, we performed six additional experiments, now buffering postsynaptic $\mathrm{Ca}^{2+}$ with BAPTA $(10 \mathrm{mM})$ in the intracellular solution. In the presence of BAPTA, trough pairing induced no significant modifications in any cell (grand average, $104 \pm 13 \%$; $n=6 ; p>0.1$ ) (Fig. $5 d$ ). Thus, induction of NMDA receptorindependent LTD required a rise in postsynaptic $\mathrm{Ca}^{2+}$.

\section{Role of the oscillatory $V_{\mathrm{m}}$ modulation}

To examine whether the imposed $V_{\mathrm{m}}$ oscillations played a specific role in the induction of synaptic modifications, we evoked postsynaptic discharges, not with sinusoidally modulated currents but by brief current pulses, the duration of which was chosen to be the same as in previous experiments on STDP $(5 \mathrm{msec}$, 400-700 pA) (supplemental Fig. 2; supplemental material, available at www.jneurosci.org). In P22 slices kept in control solution, both coincident and antiphase pairing induced significant LTD in 4 of 10 and 3 of 6 cases, respectively (grand averages, coincident: $83 \pm 6 \%, n=10, p<0.001$; antiphase pairing: $86 \pm 11 \%$, $n=6, p<0.001$ ). Even with carbachol added, both coincident and antiphase pairing continued to induce LTD in nine of nine and two of five experiments, respectively (grand averages, coincident pairing: $59 \pm 8 \%, n=9, p<0.001$; antiphase pairing: $90 \pm 16 \%, n=5, p<0.05$ ) (supplemental Fig. 2; supplemental material, available at www.jneurosci.org). These results suggest that $V_{\mathrm{m}}$ oscillations enhance the phase sensitivity of synaptic modifications. 


\section{Increased conductance state}

To match as closely as possible highconductance conditions observed in vivo when neurons engage in synchronized $\beta$ and $\gamma$ oscillations, we repeated $40 \mathrm{~Hz}$ carbachol experiments in older rats (P2531), now adding $200 \mathrm{~nm}$ kainate a few minutes before and during pairing $(n=$ 12). The combined application of carbachol and nanomolar doses of kainate has been used as a tool to induce network oscillations in the $\gamma$ frequency range in cortical preparations in vitro (Buhl et al., 1998). We wanted to induce a highconductance state without network oscillations, however, because they would interfere with the precision of our pairing protocol. Application of kainate resulted in a drop of the input resistance of the cells (drop of $-16 \pm 4 \%$; $n=12$ cells) associated with increased synaptic background activity and an acceleration of the decay of the electrically evoked EPSPs (Fig. 6, inset), but the spontaneous $V_{\mathrm{m}}$ fluctuations showed no evidence of an oscillatory modulation. Power spectra before and during kainate application revealed no significant change in any frequency band $(1-4,4-8,8-14,14-20,20-48 \mathrm{~Hz}$; data not shown). In this state of increased synaptic conductance, $40 \mathrm{~Hz}$ oscillation peak pairing led to LTP in four of six experiments, whereas trough pairing led to LTD in four of six cells; the remaining cells showed no significant change (Fig. 6a). LTP was first revealed after complete wash of kainate but resembled the LTP observed in the carbachol experiments without kainate. The grand averages of both peak and trough pairing effects revealed highly significant LTP and LTD, respectively (grand averages, peak: $119 \pm 14 \%, n=6, \mathrm{p} \ll 0.001$; trough: $70 \pm 9 \%, n=$ $6, \mathrm{p} \ll 0.001$ ) (Fig. 6b). Thus, even in a state of increased conductance, synaptic modifications among fast-oscillating cells remain exquisitely phase sensitive.

\section{Discussion}

A phase dependency of synaptic modifications has been reported previously in the hippocampus for slow network oscillations in the $\theta$ range [ $5-8 \mathrm{~Hz}$, in vitro (Huerta and Lisman, 1993, 1995), in vivo (Hölscher et al., 1997)]. In these studies, synaptic strength was assessed from field potentials. These were found to undergo potentiation after pairing of electrically evoked afferent volleys with oscillation peaks, and this potentiation could be reset by subsequent trough pairing. No evidence was obtained that LTD could be induced de novo by trough pairing. The present results extend these experiments to the neocortex and demonstrate with intracellular recordings that synaptic modifications remain highly sensitive to the phase relation between periodic presynaptic and postsynaptic activity even if presynaptic and postsynaptic elements engage in sustained activity in the high-frequency range of $\beta$ and $\gamma$ oscillations. This phase sensitivity depended on the oscillatory $V_{\mathrm{m}}$ modulation of the postsynaptic cell on NMDA receptors and in slices of older rats on the presence of cholinergic agonists. The phase sensitivity of synaptic modifications (con-
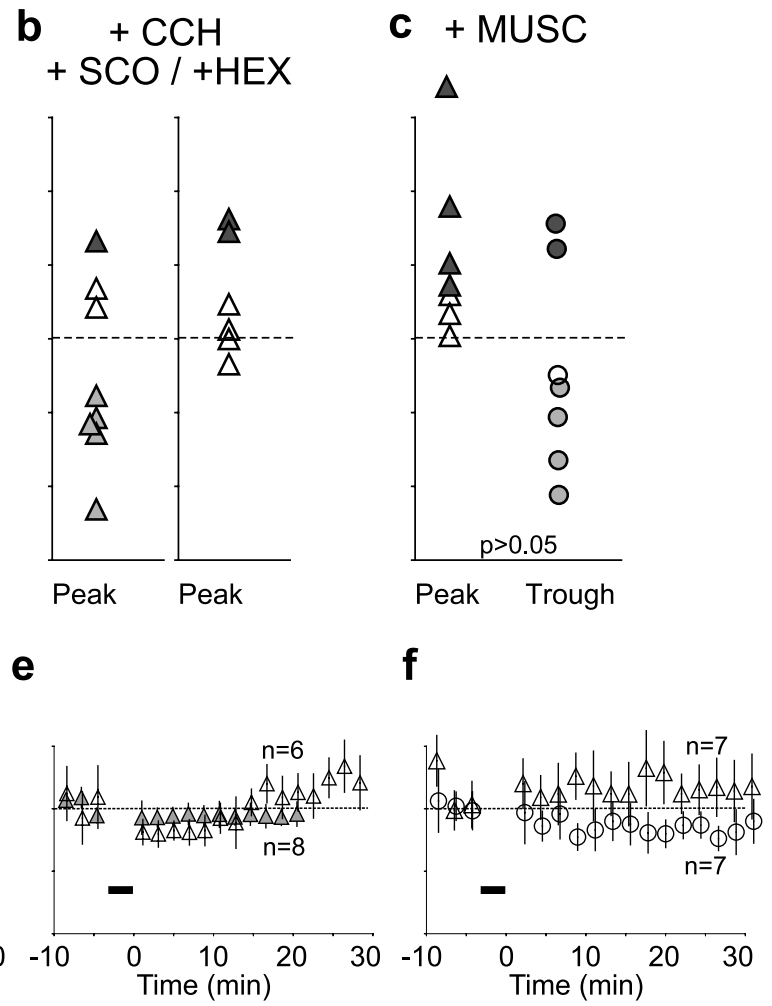

Figure 4. Synaptic modifications resulting from peak and trough pairing in different cholinergic pharmacological conditions in carbachol data are depicted in gray. $f$, Time course of EPSP amplitude changes in the presence of muscarine (mean \pm SD). Triangles indicate peak pairing; circles indicate trough pairing.

trast between effects of peak and trough pairing) still persisted during increased network activity driven by kainate application.

In the present experiments, peak pairing led to NMDA receptor-dependent LTP and trough pairing led to NMDA receptor-independent LTD. In contrast to slices of young rats, LTP induction in slices of older rats required activation of muscarinic receptors, suggesting that carbachol effects had compensated for the age-dependent elevation of the LTP threshold. Evidence suggests that this elevation is caused in part by the maturation of inhibitory mechanisms (Kato et al., 1991) and changes in the subunit composition of NMDA receptors (Nase et al., 2003). Carbachol had caused a slight depolarization of resting $V_{\mathrm{m}}$ and a reduction of spike-frequency adaptation (data not shown), probably because of a blockade of $\mathrm{K}^{+}$currents $\left(I_{\mathrm{AHP}}, I_{\mathrm{M}}\right)$ (Nicoll et al., 1990). This could have enhanced LTP by increasing dendritic depolarization and facilitating backpropagation of action potentials. Other nonexclusive possibilities are that carbachol enhanced NMDA receptor efficiency directly (Kirkwood et al., 1999) and/or increased $\mathrm{Ca}^{2+}$ release from endogenous stores, both effects being mediated via the $\mathrm{IP}_{3}$ pathway (Markram and Segal, 1992; Nakamura et al., 2000). With peak pairing, activation of muscarinic receptors with carbachol or muscarine had increased the incidence of LTP. This is best explained by assuming a lowering of the threshold that separates the activation levels required for the induction of LTD and LTP (Artola and Singer, 1987; Artola et al., 1990; Hansel et al., 1997).

LTP facilitation was found to depend on the activation of muscarinic receptors; however, a contribution of nicotinic recep- 

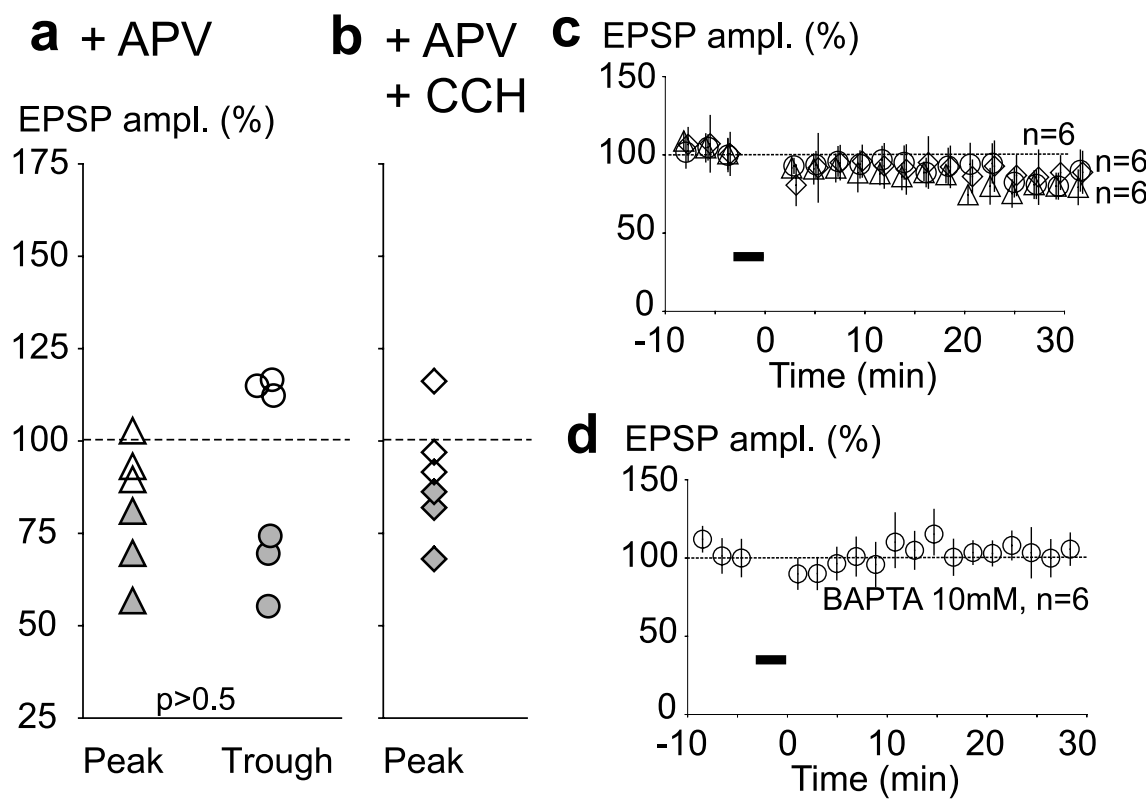

Figure 5. Synaptic modifications resulting from peak and trough pairing during NMDA receptor and intracellular $\mathrm{Ca}^{2+}$ blockade in P22 rats. a, APV. Symbols are the same as in Figure 2a.b, APV and carbachol.c, Time course of EPSP amplitude changes with NMDA receptor blockade (mean $\pm S D$ ). Triangles indicate peak pairing; circles indicate trough pairing; diamonds indicate peak pairing in the presence of APV and carbachol. $d$, Time course of EPSP amplitude changes with intracellular BAPTA (mean \pm $\mathrm{SD})$. Circles indicate trough pairing.

tors might be considered, because the LTD observed in carbachol after blockade of muscarinic receptors was much weaker than the LTD obtained in control solution and after blockade of both receptor subtypes. The balanced activation of both muscarinic and nicotinic receptor subtypes might act synergistically to support the phase sensitivity of synaptic plasticity during fast oscillations.

Trough pairing never induced LTP in either P13 or P22 slices treated with carbachol and kainate, suggesting that this protocol provides robust conditions for LTD induction. This is remarkable in view of the sustained high-frequency discharges (20 or 40 $\mathrm{Hz}$ ) imposed on both presynaptic and postsynaptic elements and the high affinity of NMDA receptors for glutamate (see below).

The role of the $V_{\mathrm{m}}$ oscillations appeared to be more than a clocking device to time action potentials, because coincident step-pulse pairing induced LTD and failed to induce LTP despite the presence of carbachol (supplemental material, available at www.jneurosci.org). Because we did obtain LTP in the experiments with oscillatory $V_{\mathrm{m}}$ modulation, all other conditions being identical, we conclude that the susceptibility to undergo synaptic modifications differs in regimens in which cells undergo an oscillatory modulation of their $V_{\mathrm{m}}$ and in which they are just driven above threshold by brief current injections. We propose the following possibilities. First, sinusoidal currents are likely to result in larger dendritic depolarization than brief current pulses because of dendritic low-pass filtering. Sinusoidal currents therefore should be more efficient in facilitating activation of NMDA receptors and voltage-dependent calcium channels and backpropagation of spikes (Stuart and Häusser, 2001). Second, with sinusoidal $V_{\mathrm{m}}$ oscillations, the $V_{\mathrm{m}}$ of the cells occupies depolarized levels over longer time than with step pulses. With peak pairing, EPSPs occurred in the middle of the depolarizing stretches, which should provide particularly favorable conditions for prolonged activation of NMDA receptor-mediated $\mathrm{Ca}^{2+}$ entry. Third, the hyperpolarizing phases of $V_{\mathrm{m}}$ oscillations could have favored deinactivation of previously activated, voltage- gated $\mathrm{Na}^{+}$and $\mathrm{Ca}^{2+}$ channels, which could have contributed to a stronger depolarization with peak pairing.

The conditions of our experiments (recordings at physiological temperature with intact inhibition, synchronized EPSP multifiber volleys exhibiting an oscillatory modulation at $20 \mathrm{~Hz}$; oscillatory modulation of the $V_{\mathrm{m}}$ of postsynaptic cells at depolarized $V_{\mathrm{m}}$ levels associated with periodic firing at 20 or $40 \mathrm{~Hz}$; stimulation of cholinergic and kainate receptors) closely resemble those in vivo when neuronal networks engage in synchronized gamma oscillations (Gray and McCormick, 1996; Azouz and Gray, 2000). The major difference is that in the present experiments $V_{\mathrm{m}}$ oscillations were induced with current injection, whereas in vivo they are attributable to the alternation of synchronized volleys of EPSPs and IPSPs (Bringuier et al., 1997). Hence, in vivo, oscillations are associated with periodic increases of membrane conductance and shortened membrane time constants (Traub et al., 1996; Lampl et al., 1999). We predict from this that the phase sensitivity of synaptic modifications should be even higher in vivo than under the present conditions, when neuronal networks engage in highfrequency oscillations. This prediction is supported by the finding that the differences between the effects of peak and trough pairing persisted in a kainate-driven increased conductance state.

Unlike in previous studies on STDP, we evoked periodic discharge patterns in both presynaptic and postsynaptic elements, imposed an oscillatory modulation of $V_{\mathrm{m}}$ on the postsynaptic cells, and varied the phase angles between the periodic discharge sequences between in-phase and of-phase conditions. In the first case, EPSPs were coincident with postsynaptic discharges and the peaks of $V_{\mathrm{m}}$ depolarizations; in the second, they were equidistant from the preceding and following spikes and fell in the troughs of the $V_{\mathrm{m}}$ oscillations. Under these conditions, causal relationships between presynaptic and postsynaptic responses are no longer clearly defined. Still, the changes in phase angle led to a reversal of the polarity of synaptic changes, suggesting that the Hebbian LTP mechanism evaluates not only causal relations between presynaptic and postsynaptic discharges, as demonstrated in STDP experiments, but also the synchronicity between oscillatory presynaptic and postsynaptic activity. We propose that the mechanisms are the same as those supporting STDP. Peak pairing leads to maximal cooperativity between presynaptic and postsynaptic activity even if EPSPs jitter around the interval of postsynaptic spiking, because EPSPs coincide with phases of maximal $V_{\mathrm{m}}$ depolarization and postsynaptic spikes. Both factors increase $\mathrm{Ca}^{2+}$ entry through NMDA receptors, which was a necessary prerequisite for LTP induction in the present experiments, and in addition, both enhance the probability of $\mathrm{Ca}^{2+}$ entry through voltage-gated $\mathrm{Ca}^{2+}$ channels. In contrast, with trough pairing, EPSPs are less likely to activate NMDA receptor-gated $\mathrm{Ca}^{2+}$ channels, so that synaptic modifications, in this case LTD, should depend mainly on voltage-gated $\mathrm{Ca}^{2+}$ channels and/or metabotropic glutamate receptors. This conjecture is supported by the finding that blockade of NMDA receptors had no effect on the incidence of trough 


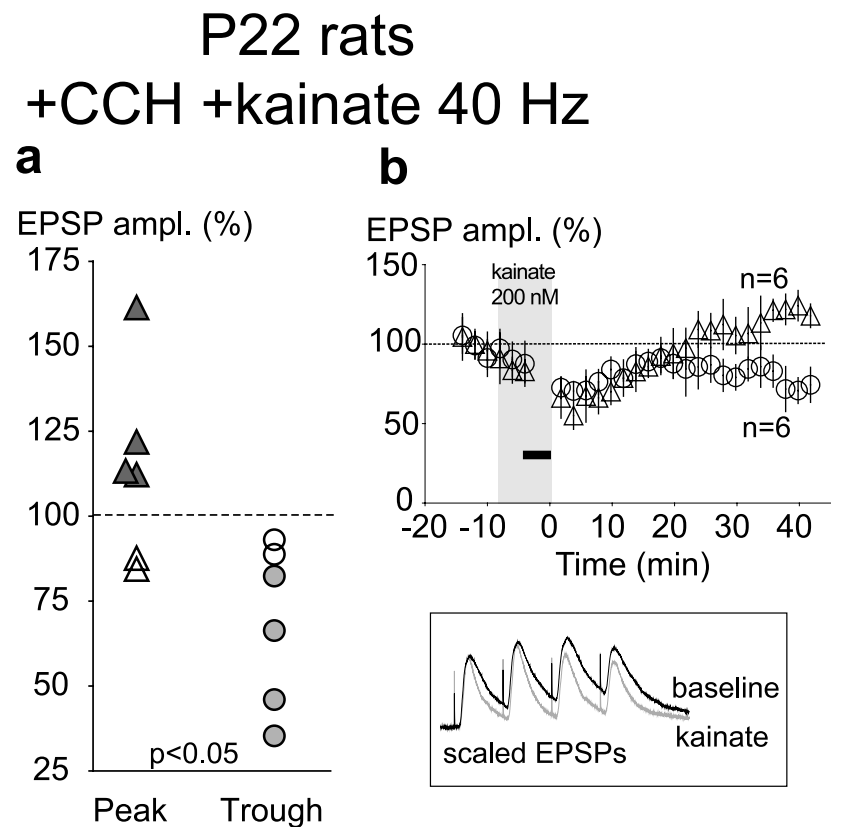

Figure 6. Phase sensitivity of synaptic modifications during sustained high-frequency oscillations, cholinergic receptor activation, and increased conductance state. $a, b, \mathrm{P} 22$ rats in $\mathrm{CCH}$ and kainate with $40 \mathrm{~Hz}$ oscillations. $a$, Synaptic modifications after peak and trough pairing. Each symbol corresponds to one experiment and represents the pairing-induced change of the first test EPSP averaged across 20 measurements taken before application of kainate and 3540 min after pairing. Black symbols represent significant LTP, gray symbols represent significant LTD, and empty symbols represent no significant changes. $P$ value refers to the difference between peak and trough pairing (Mann-Whitney rank sum test for all experiments). $b$, Corresponding time course of EPSP amplitude changes (mean \pm SD). Inset, Representtive test responses collected before and during application of $200 \mathrm{nM}$ kainate. Traces represent the average of 10 consecutive measurements. Note the faster EPSP decay during kainate-driven increased conductance.

pairing-induced LTD and that LTD was also inducible with presynaptic stimulation alone.

The question remains, however, of why NMDA receptor activation contributed so little to the effects of trough pairing, especially when cells oscillated at $40 \mathrm{~Hz}$. In this case, EPSPs peaked $\sim 12$ msec before the respective next spikes. In STDP experiments, such a condition leads to NMDA receptor activation and to LTP, especially when discharge frequencies are high (Sjöström et al., 2001). The most likely interpretation is that in the present experiments the contribution of NMDA receptors was reduced by inhibition and by the oscillatory $V_{\mathrm{m}}$ modulation that caused rapid succession of hyperpolarizing phases. We could exclude the possibility that the test stimuli used for the assessment of synaptic gain changes evoked monosynaptic IPSPs that could have contaminated the test EPSPs (see Materials and Methods), but it is very likely that the high-frequency trains used for the induction of modifications recruited polysynaptic inhibition. Thus, inhibition evoked by the trough stimuli was probably still effective around the peak of the afterdepolarization cycle. We propose that this reduced the probability of lifting the $\mathrm{Mg}^{2+}$ block of NMDA receptors that are still occupied by glutamate released by the trough stimuli. In addition, because of the high-frequency oscillations, the time window for the removal of the $\mathrm{Mg}^{2+}$ block was short because of the intervening hyperpolarization phases. Both mechanisms are likely to sharpen the phase sensitivity of the synaptic modifications by focusing the window for LTP induction on the peaks of the $V_{\mathrm{m}}$ oscillations.

The present results indicate that connections between oscillating cell groups strengthen or weaken depending on whether the cells oscillate in phase or in antiphase. Such a mechanism establishes associations between cells exhibiting synchronous firing patterns, regardless of the origins of correlated firing. Precisely synchronized firing can have several causes. It can result from stimulus locking of responses to temporally contingent stimuli (Rager and Singer, 1998), it can be caused by common input from diverging afferents, or it can be caused by dynamic, selforganizing interactions among reciprocally coupled neurons. In the latter case, the synchronizing effects are mediated by both excitatory and inhibitory interactions (Buhl et al., 1998), which are mediated by reciprocal corticocortical connections in the neocortex. A mechanism that enhances coupling between synchronously active cell groups this is ideally suited to support associative learning and the formation of cell assemblies. It can establish associations as a function of temporally contingent external events, but it can also associate neurons that have synchronized their responses because of internal grouping and binding operations.

Of particular relevance for functional interpretations is the finding that synaptic plasticity remained sensitive to small shifts in synchrony even when cells engaged in sustained, periodic, high-frequency discharges such as occur during $\gamma$ oscillations in vivo. This suggests that precise synchrony of discharges serves as a signature of relatedness in Hebbian LTP. An important implication is that precise synchrony is then likely to also serve as a tag of relatedness in signal processing. The reason is that learning of false or spurious conjunctions can be avoided only if the code that defines neuronal responses as related operates on exactly the same time scale in signal processing and synaptic plasticity.

\section{References}

Alonso A, de Curtis M, Llinas R (1990) Postsynaptic Hebbian and nonHebbian long-term potentiation of synaptic efficacy in the entorhinal cortex in slices and in the isolated adult guinea pig brain. Proc Natl Acad Sci USA 87:9280-9284.

Artola A, Singer W (1987) Long-term potentiation and NMDA receptors in rat visual cortex. Nature 330:649-652.

Artola A, Bröcher S, Singer W (1990) Different voltage-dependent thresholds for inducing long-term depression and long-term potentiation in slices of rat visual cortex. Nature 347:69-72.

Azouz R, Gray CM (2000) Dynamic spike threshold reveals a mechanism for synaptic coincidence detection in cortical neurons in vivo. Proc Natl Acad Sci USA 97:8110-8115.

Bear MF, Kirkwood A (1993) Neocortical long-term potentiation. Curr Opin Neurobiol 3:197-202.

Bringuier V, Fregnac Y, Baranyi A, Debanne D, Shulz DE (1997) Synaptic origin and stimulus dependency of neuronal oscillatory activity in the primary visual cortex of the cat. J Physiol (Lond) 500:751-774.

Bröcher S, Artola A, Singer W (1992) Agonists of cholinergic and noradrenergic receptors facilitate synergistically the induction of long-term potentiation in slices of rat visual cortex. Brain Res 573:27-36.

Buhl EH, Tamas G, Fisahn A (1998) Cholinergic activation and tonic excitation induce persistent gamma oscillations in mouse somatosensory cortex in vitro. J Physiol (Lond) 513:117-126.

Buzsaki G, Chrobak JJ (1995) Temporal structure in spatially organized neuronal ensembles: a role for interneuronal networks. Curr Opin Neurobiol 5:504-510.

Celesia GG, Jasper HH (1966) Acetylcholine released from cerebral cortex in relation to state of activation. Neurology 16:1053-1063.

Christofi G, Nowicky AV, Bolsover SR, Bindman LJ (1993) The postsynaptic induction of non-associative long-term depression of excitatory synaptic transmission in rat hippocampal slices. J Neurophysiol 69:219-229.

Cossart R, Aronov D, Yuste R (2003) Attractor dynamics of network UP states in the neocortex. Nature 423:283-288.

Deuchars J, West DC, Thomson AM (1994) Relationships between morphology and physiology of pyramid-pyramid single axon connections in rat neocortex in vitro. J Physiol (Lond) 478:423-435.

Egger V, Feldmeyer D, Sakmann B (1999) Coincidence detection and 
changes of synaptic efficacy in spiny stellate neurons in rat barrel cortex. Nat Neurosci 2:1098-1105.

Engel AK, Fries P, Singer W (2001) Dynamic predictions: oscillations and synchrony in top-down processing. Nat Rev Neurosci 2:704-716.

Feldman DE (2000) Timing-based LTP and LTD at vertical inputs to layer II/III pyramidal cells in rat barrel cortex. Neuron 27:45-56.

Fernandez G, Effern A, Grunwald T, Pezer N, Lehnertz K, Dumpelmann M, Van Roost D, Elger CE (1999) Real-time tracking of memory formation in the human rhinal cortex and hippocampus. Science 285:1582-1585.

Fries P, Neuenschwander S, Engel AK, Goebel R, Singer W (2001) Rapid feature selective neuronal synchronization through correlated latency shifting. Nat Neurosci 4:194-200.

Froemke RC, Dan Y (2002) Spike-timing-dependent synaptic modification induced by natural spike trains. Nature 416:433-438.

Gray CM (1999) The temporal correlation hypothesis of visual feature integration: still alive and well. Neuron 24:31-47, 111-125.

Gray CM, McCormick DA (1996) Chattering cells: superficial pyramidal neurons contributing to the generation of synchronous oscillations in the visual cortex. Science 274:109-113.

Hansel C, Artola A, Singer W (1997) Relation between dendritic $\mathrm{Ca}^{2+}$ levels and the polarity of synaptic long-term modifications in rat visual cortex neurons. Eur J Neurosci 9:2309-2322.

Hestrin S (1992) Developmental regulation of NMDA receptor-mediated synaptic currents at a central synapse. Nature 357:686-689.

Hölscher C, Anwyl R, Rowan MJ (1997) Stimulation on the positive phase of hippocampal theta rhythm induces long-term potentiation that can be depotentiated by stimulation on the negative phase in area CA1 in vivo. J Neurosci 17:6470-6477.

Huerta PT, Lisman JE (1993) Heightened synaptic plasticity of hippocampal CA1 neurons during a cholinergically induced rhythmic state. Nature 364:723-725.

Huerta PT, Lisman JE (1995) Bidirectional synaptic plasticity induced by a single burst during cholinergic theta oscillation in CA1 in vitro. Neuron 15:1053-1063.

Kato N, Artola A, Singer W (1991) Developmental changes in the susceptibility to long-term potentiation of neurones in rat visual cortex slices. Brain Res Dev Brain Res 60:43-50.

Kirkwood A, Rozas C, Kirkwood J, Perez F, Bear MF (1999) Modulation of long-term synaptic depression in visual cortex by acetylcholine and norepinephrine. J Neurosci 19:1599-1609.

Lampl I, Reichova I, Ferster D (1999) Synchronous membrane potential fluctuations in neurons of the cat visual cortex. Neuron 22:361-374.

Markram H, Segal M (1992) The inositol 1,4,5-trisphosphate pathway mediates cholinergic potentiation of rat hippocampal neuronal responses to NMDA. J Physiol (Lond) 447:513-533.

Markram H, Tsodyks M (1996) Redistribution of synaptic efficacy between neocortical pyramidal neurons. Nature 382:807-810.

Markram H, Lübke J, Frotscher M, Sakmann B (1997) Regulation of synaptic efficacy by coincidence of postsynaptic APs and EPSPs. Science 275:213-215.

Marrosu F, Portas C, Mascia MS, Casu MA, Fa M, Giagheddu M, Imperato A, Gessa GL (1995) Microdialysis measurement of cortical and hippocampal acetylcholine release during sleep-wake cycle in freely moving cats. Brain Res 671:329-332.
Miltner WH, Braun C, Arnold M, Witte H, Taub E (1999) Coherence of gammaband EEG activity as a basis for associative learning. Nature 397:434-436.

Nakamura T, Nakamura K, Lasser-Ross N, Barbara JG, Sandler VM, Ross WN (2000) Inositol 1,4,5-trisphosphate (IP3)-mediated $\mathrm{Ca}^{2+}$ release evoked by metabotropic agonists and backpropagating action potentials in hippocampal CA1 pyramidal neurons. J Neurosci 20:8365-8376.

Nase G, Weishaupt J, Stern P, Singer W, Monyer H (1999) Genetic and epigenetic regulation of NMDA receptor expression in the rat visual cortex. Eur J Neurosci 11:4320-4326.

Nase G, Singer W, Monyer H, Engel AK (2003) Features of neuronal synchrony in mouse visual cortex. J Neurophysiol 90:1115-1123.

Nicoll RA, Malenka RC, Kauer JA (1990) Functional comparison of neurotransmitter receptor subtypes in mammalian central nervous system. Physiol Rev 70:513-565.

Parkhurst D, Law K, Niebur E (2002) Modeling the role of salience in the allocation of overt visual attention. Vision Res 42:107-123.

Quinlan EM, Philpot BD, Huganir RL, Bear MF (1999) Rapid, experiencedependent expression of synaptic NMDA receptors in visual cortex in vivo. Nat Neurosci 2:352-357.

Rager G, Singer W (1998) The response of cat visual cortex to flicker stimuli of variable frequency. Eur J Neurosci 10:1856-1877.

Rodriguez R, Kallenbach U, Singer W, Munk MHJ (2001) Reduction of gamma-oscillatory responses after scopolamine application in cat visual cortex. Soc Neurosci Abstr 27:123.3.

Shu Y, Hasenstaub A, McCormick DA (2003) Turning on and off recurrent balanced cortical activity. Nature 423:288-293.

Silva LR, Amitai Y, Connors BW (1991) Intrinsic oscillations of neocortex generated by layer 5 pyramidal neurons. Science 251:432-435.

Singer W (1993) Synchronization of cortical activity and its putative role in information processing and learning. Annu Rev Physiol 55:349-374.

Singer W (1999) Neuronal synchrony: a versatile code for the definition of relations? Neuron 24:49-65, 111-125.

Sjöström PJ, Turrigiano GG, Nelson SB (2001) Rate, timing, and cooperativity jointly determine cortical synaptic plasticity. Neuron 32:1149-1164.

Steriade M (1999) Coherent oscillations and short-term plasticity in corticothalamic networks. Trends Neurosci 22:337-345.

Steriade M, Timofeev I, Durmuller N, Grenier F (1998) Dynamic properties of corticothalamic neurons and local cortical interneurons generating fast rhythmic (30-40 Hz) spike bursts. J Neurophysiol 79:483-490.

Stuart GJ, Häusser M (2001) Dendritic coincidence detection of EPSPs and action potentials. Nat Neurosci 4:63-71.

Tallon-Baudry C, Bertrand O (1999) Oscillatory gamma activity in humans and its role in object representation. Trends Cogn Sci 3:151-162.

Traub RD, Whittington MA, Colling SB, Buzsaki G, Jefferys JG (1996) Analysis of gamma rhythms in the rat hippocampus in vitro and in vivo. J Physiol (Lond) 493:471-484.

Usrey WM, Reid RC (1999) Synchronous activity in the visual system. Annu Rev Physiol 61:435-456.

Varela F, Lachaux JP, Rodriguez E, Martinerie J (2001) The brainweb: phase synchronization and large-scale integration. Nat Rev Neurosci 2:229-239.

Volgushev M, Voronin LL, Chistiakova M, Singer W (1997) Relations between long-term synaptic modifications and paired-pulse interactions in the rat neocortex. Eur J Neurosci 9:1656-1665. 